TRANSACTIONS OF THE

AMERICAN MATHEMATICAL SOCIETY

Volume 351, Number 10, Pages 3915-3934

S 0002-9947(99)02421-6

Article electronically published on May 21, 1999

\title{
RATES OF CONVERGENCE OF DIFFUSIONS WITH DRIFTED BROWNIAN POTENTIALS
}

\author{
YUEYUN HU, ZHAN SHI, AND MARC YOR
}

\begin{abstract}
We are interested in the asymptotic behaviour of a diffusion process with drifted Brownian potential. The model is a continuous time analogue to the random walk in random environment studied in the classical paper of Kesten, Kozlov, and Spitzer. We not only recover the convergence of the diffusion process which was previously established by Kawazu and Tanaka, but also obtain all the possible convergence rates. An interesting feature of our approach is that it shows a clear relationship between drifted Brownian potentials and Bessel processes.
\end{abstract}

\section{INTRODUCTION}

Modelling a class of random phenomena in physics and biology (cf. Bouchaud et al. [4]), the simple Random Walk in Random Environment (RWRE) is defined as follows: Let $\Xi \stackrel{\text { def }}{=}\left\{\xi_{i}\right\}_{i \in \mathbf{Z}}$ be a sequence of independent and identically distributed random variables taking values in $(0,1)$, and any realization of $\Xi$ is labelled as "environment". Define the RWRE $\left\{S_{n}\right\}_{n \geq 0}$ by $S_{0}=0$ and

$$
\mathbf{P}\left(S_{n+1}=j \mid S_{n}=i ; \Xi\right)= \begin{cases}\xi_{i}, & \text { if } j=i+1, \\ 1-\xi_{i}, & \text { if } j=i-1, \\ 0, & \text { otherwise, }\end{cases}
$$

for any $n \geq 1$ and $i \in \mathbf{Z}$. Among the best known works on RWRE, Solomon [30] treats the basic recurrence/transience problem, Sinai [29] and Kesten et al. [21] establish limit theorems respectively in recurrent and transient situations, Deheuvels and Révész [9] investigate the almost sure behaviour in Sinai's setting, and Csörgö et al. [8] study the local time. We refer to the books of Révész [25] and Hughes [16] for a detailed account. There is also a renewed interest concerning various aspects of RWRE. Let us mention recent papers by Alili [1], Comets et al. [7], Dembo et al. [10], Gantert and Zeitouni [12], Greven and den Hollander [13], Hu and Shi [14]-[15], and Shi [28].

In the recurrent case treated in the celebrated paper of Sinai [29], it is shown that RWRE moves far slower than the usual random walk (in non-random environment). On the other hand, the convergence and rates of RWRE in the transient case are completely characterized by Kesten et al. [21].

In this paper, we are interested in the continuous time analogue of RWRE, namely, a diffusion process with random potential, introduced by Schumacher [27]

Received by the editors November 17, 1997 and, in revised form, July 3, 1998.

1991 Mathematics Subject Classification. Primary 60J60, 60F05.

Key words and phrases. Diffusion with random potential, Bessel process, rate of convergence. 
and Brox [5]. In what follows, $\{W(x) ; x \in \mathbf{R}\}$ denotes a two-sided Brownian motion with $W(0)=0$, and

$$
V(x) \stackrel{\text { def }}{=} W(x)-\frac{\kappa}{2} x, \quad x \in \mathbf{R},
$$

is a Brownian motion with drift $(-\kappa / 2)$. Consider the formal solution of the following stochastic differential equation:

$$
\left\{\begin{aligned}
\mathrm{d} X(t) & =\mathrm{d} \beta(t)-\frac{1}{2} V^{\prime}(X(t)) \mathrm{d} t \\
X(0) & =0
\end{aligned}\right.
$$

where $\beta$ denotes a Brownian motion starting from 0 , independent of $W$ (hence of the random potential $V)$. We note that changing $\kappa$ into $(-\kappa)$ leads to the same formal equation for $(-X)$, since $\{-\beta(t) ; t \geq 0\}$ and $\{W(-x) ; x \in \mathbf{R}\}$ are independent Brownian motions. Hence, we may, without loss of generality, restrict ourselves to $\kappa \geq 0$. In fact, we shall only discuss the case $\kappa>0$, which characterizes the transience of $X$ (see Brox [5] for the recurrence with $\kappa=0$, and Kawazu and Tanaka [19] for the transience with $\kappa>0$ ).

Rigorously speaking, instead of writing the formal derivative of $V$ in (1.2), we should consider $X$ as a diffusion process with generator

$$
\frac{1}{2} \mathrm{e}^{V(x)} \frac{\mathrm{d}}{\mathrm{d} x}\left(\mathrm{e}^{-V(x)} \frac{\mathrm{d}}{\mathrm{d} x}\right) .
$$

A representation of $\{X(t) ; t \geq 0\}$ using time change is given in Section 3. We refer to Kawazu and Tanaka [19], Mathieu [23], and Carmona [6] for related studies and further extensions.

This paper aims at completing previous work of Kawazu and Tanaka [19], [20], which respectively obtain the convergence of $X$, and its convergence rate in the particular case $\kappa>2$. Our main result is a complete characterization of all possible convergence rates, therefore providing the continuous time analogue of what Kesten et al. [21] established for transient RWRE. Our approach is new, mainly based on Bessel process techniques by showing how Bessel and Jacobi processes can be used in the study of random potentials.

To state our result, we define

$$
H(r) \stackrel{\text { def }}{=} \inf \{t>0: X(t)>r\}, \quad r>0,
$$

the first hitting time process associated with $X$. Throughout the paper, $\mathcal{N}$ stands for a Gaussian $\mathcal{N}(0,1)$ variable, $\mathcal{S}_{\alpha}^{\text {ca }}$ (for $0<\alpha<2$ ) a completely asymmetric stable variable of index $\alpha$, and $\mathcal{C}_{\lambda}^{\text {ca }}$ (for $\lambda>0$ ) a (positive) completely asymmetric Cauchy variable of parameter $\lambda$ (the superscript "ca" standing for "completely asymmetric"). When $0<\alpha<1, \mathcal{S}_{\alpha}^{\text {ca }}$ is a positive variable. The distributions of $\mathcal{S}_{\alpha}^{\mathrm{ca}}$ and $\mathcal{C}_{\lambda}^{\mathrm{ca}}$ are characterized by their respective characteristic functions: for $t \in \mathbf{R}$,

$$
\begin{aligned}
& \mathbf{E} \exp \left(i t \mathcal{S}_{\alpha}^{\mathrm{ca}}\right)=\exp \left[-|t|^{\alpha}\left(1-i \operatorname{sgn}(t) \tan \left(\frac{\pi \alpha}{2}\right)\right)\right], \\
& \mathbf{E} \exp \left(i t \mathcal{C}_{\lambda}^{\mathrm{ca}}\right)=\exp \left[-\lambda\left(|t|+i t \frac{2}{\pi} \log |t|\right)\right] .
\end{aligned}
$$

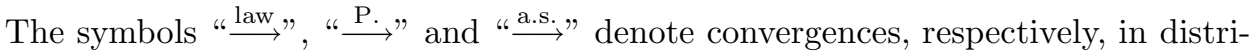
bution, in probability and with probability one. 
Throughout the paper, we write

$$
\begin{aligned}
c_{0} & \stackrel{\text { def }}{=} 2 \gamma+\log \left(\frac{\pi}{4}\right), \\
\psi(x) & \stackrel{\text { def }}{=}\left(\frac{\pi x}{4 \Gamma^{2}(x) \sin (\pi x / 2)}\right)^{1 / x}, \quad 0<x<2,
\end{aligned}
$$

which are featured in several constants below ( $\gamma$ denoting Euler's constant, and $\Gamma$ the usual gamma function).

Theorem A (Kawazu and Tanaka [19]). As r tends to infinity,

$$
\begin{aligned}
& \frac{H(r)}{r^{1 / k}} \stackrel{\text { law }}{\longrightarrow} c_{1} \mathcal{S}_{\kappa}^{\mathrm{ca}}, \quad 0<\kappa<1, \\
& \frac{H(r)}{r \log r} \stackrel{\mathrm{P} .}{\longrightarrow} 4, \quad \kappa=1, \\
& \frac{H(r)}{r} \stackrel{\text { a.s. }}{\longrightarrow} \frac{4}{\kappa-1}, \quad \kappa>1,
\end{aligned}
$$

where $c_{1}=c_{1}(\kappa)>0$ is a finite constant depending on $\kappa$ whose value is given in Section 3 .

Here is the main result of the paper, characterizing all possible convergence rates of $H$.

Theorem 1.1. For $\kappa \geq 1$, the following central limit results hold, as $r$ tends to infinity:

$$
\begin{aligned}
\frac{1}{r}(H(r)-4 r \log r) & \stackrel{\text { law }}{\longrightarrow} 4\left(c_{0}-1-2 \log 2\right)+\frac{\pi}{4} \mathcal{C}_{8}^{\mathrm{ca}}, \quad \kappa=1, \\
\frac{1}{r^{1 / \kappa}}\left(H(r)-\frac{4}{\kappa-1} r\right) & \stackrel{\text { law }}{\longrightarrow} 8 \psi(\kappa) \mathcal{S}_{\kappa}^{\mathrm{ca}}, \quad 1<\kappa<2, \\
\frac{1}{(r \log r)^{1 / 2}}\left(H(r)-\frac{4}{\kappa-1} r\right) & \stackrel{\text { law }}{\longrightarrow} 8 \mathcal{N}, \quad \kappa=2, \\
\frac{1}{r^{1 / 2}}\left(H(r)-\frac{4}{\kappa-1} r\right) & \stackrel{\text { law }}{\longrightarrow} 8 \sqrt{\frac{\kappa}{(\kappa-1)^{3}(\kappa-2)}} \mathcal{N}, \quad \kappa>2 .
\end{aligned}
$$

Remark 1.2. As we have mentioned before, (1.13) is due to Kawazu and Tanaka [20]. Our proof of Theorem 1.1 allows us also to recover (1.7) and (1.8), with an explicit value of the constant $c_{1}$, cf. (3.13) in Section 3. Tanaka [32] also obtained (1.10)-(1.12) independently from us.

Remark 1.3. Theorem 1.1 gives a precise description of the asymptotics of $H(r)$. According to an observation going back to Kesten et al. [21], the convergence rates (1.7)-(1.13) can be transferred to $X(t)$. For more details, cf. [21], [19], or [6]. Possibly, in (1.8), the convergence holds almost surely, but our method, which uses identities in law, does not allow us to show this. We also note that in the corresponding discrete time setting, Kesten et al. [21] state the convergence in probability.

The rest of the paper is organized as follows. Section 2 is devoted to some preliminaries about Bessel processes. In Section 3, we show how Bessel processes can be applied to study random potentials. We thus provide a complete proof of Theorem 1.1. Some further discussions on principal values and stable Lévy processes are presented in Section 4. 
Notation. The usual symbol $a(x) \sim b(x)\left(x \rightarrow x_{0}\right)$ means $\lim _{x \rightarrow x_{0}} a(x) / b(x)=$ 1. We use $\operatorname{BES}(d)$ (resp. BESQ $(d)$ ) to denote a Bessel process (resp. squared Bessel process) of dimension $d$. Furthermore, $\log _{2}$ stands for the iterated logarithm function. Unless stated otherwise, $\varepsilon(\cdot)$ denotes an asymptotically negligible random term in the sense that $\varepsilon(t) \stackrel{\mathrm{P} .}{\longrightarrow} 0$ (as $t \rightarrow \infty$ ), whose value may change from one line to another. Finally, we shall also use, whenever possible, the notation $d^{\prime}$ for $d / 2$, which will often be convenient in our formulae involving dimension parameters $d_{1}$ and $d_{2}$.

Acknowledgment. We are grateful to the referee for valuable comments which helped us to improve the presentation of our paper and give more accurate references.

\section{Bessel AND JaCoBi PRocesses}

Throughout this section, $\left\{R_{1}(t) ; t \geq 0\right\}$ and $\left\{R_{2}(t) ; t \geq 0\right\}$ denote independent Bessel processes, of dimensions $d_{1}$ and $d_{2}$ respectively. We assume $R_{1}(0)=r_{1} \geq 0$ and $R_{2}(0)=r_{2}>0$. Our aim is to study the asymptotic behaviour of

$$
\Xi(t) \stackrel{\text { def }}{=} \int_{0}^{t} \frac{R_{1}^{2}(s)}{R_{2}^{4}(s)} \mathrm{d} s
$$

as $t$ tends to infinity. Observe that, in order to ensure the finiteness of $\Xi(t)$, we have to take $d_{2} \geq 2$, a hypothesis which, unless stated otherwise, we assume throughout this section.

Here is the main estimate of this section, which plays an important role in the proof of Theorem 1.1 in Section 3. Recall that $d_{1}^{\prime} \stackrel{\text { def }}{=} d_{1} / 2$ and $d_{2}^{\prime} \stackrel{\text { def }}{=} d_{2} / 2$.

Proposition 2.1. Let $d_{1}>0$ and $d_{2}>2$, and let $\Xi(t)$ be as in (2.1). When $t$ tends to infinity (writing $h(t) \stackrel{\text { def }}{=} d_{1}(\log t) /\left(d_{2}-2\right)\left(d_{2}-4\right)$ for brevity),

$$
\begin{gathered}
\frac{\Xi(t)}{(\log t)^{1 /\left(d_{2}^{\prime}-1\right)}} \stackrel{\text { law }}{\longrightarrow} c_{2} \mathcal{S}_{d_{2}^{\prime}-1}^{\mathrm{ca}}, \quad 2<d_{2}<4, \\
\frac{1}{\log t}\left(\Xi(t)-\frac{d_{1}}{4} \log t \log _{2} t\right) \stackrel{\text { law }}{\longrightarrow} c_{3}+\frac{\pi}{8\left(d_{1}+2\right)} \mathcal{C}_{d_{1}\left(d_{1}+2\right)}^{\mathrm{ca}}, \quad d_{2}=4, \\
\frac{\Xi(t)-h(t)}{(\log t)^{1 /\left(d_{2}^{\prime}-1\right)}} \stackrel{\text { law }}{\longrightarrow} c_{2} \mathcal{S}_{d_{2}^{\prime}-1}^{\mathrm{ca}}, \quad 4<d_{2}<6, \\
\frac{\Xi(t)-h(t)}{(\log t)^{1 / 2}\left(\log _{2} t\right)^{1 / 2}} \stackrel{\text { law }}{\longrightarrow} \sqrt{\frac{d_{1}\left(d_{1}+2\right)}{32}} \mathcal{N}, \quad d_{2}=6, \\
\frac{\Xi(t)-h(t)}{(\log t)^{1 / 2}} \stackrel{\text { law }}{\longrightarrow} c_{4} \mathcal{N}, \quad d_{2}>6,
\end{gathered}
$$

where

$$
\begin{aligned}
& c_{2} \stackrel{\text { def }}{=} \frac{1}{2} \psi\left(d_{2}^{\prime}-1\right)\left[\frac{8}{\left(d_{2}-2\right)^{2} B\left(d_{1}^{\prime}, d_{2}^{\prime}-1\right)}\right]^{1 /\left(d_{2}^{\prime}-1\right)}, \\
& c_{3} \stackrel{\text { def }}{=} \frac{d_{1}^{2}}{8} \int_{0}^{1} y^{d_{1}^{\prime}-1} \log (1-y) \mathrm{d} y+\frac{d_{1}\left(c_{0}-\log \left(d_{1}+2\right)\right)}{4}, \\
& c_{4} \stackrel{\text { def }}{=} \frac{d_{1}}{\left(d_{2}^{\prime}-1\right)\left(d_{2}-4\right)}\left[\frac{1}{d_{2}-2}+\frac{1}{d_{2}-4}+\frac{2}{d_{2}-6}+\frac{d_{2}-2}{d_{1}\left(d_{2}-6\right)}\right]^{1 / 2},
\end{aligned}
$$

and $B(\cdot, \cdot)$ denotes the beta function. 
Remark 2.2. We shall not use (2.6) in the paper. It is presented here only for the sake of completeness. It is also possible to treat the case $d_{1}>0, d_{2}=2$, for which one can prove that

$$
\frac{\log \Xi(t)}{\log t} \stackrel{\text { law }}{\longrightarrow} \frac{1}{\mathcal{E}}, \quad t \rightarrow \infty,
$$

where $\mathcal{E}$ denotes an exponential variable with mean 1 .

The proof of Proposition 2.1 relies on some preliminaries, stated below as Lemmas 2.3 and 2.8 .

Lemma 2.3. Let $\beta$ be a standard Brownian motion, with local time $\left\{L_{t}^{x} ; t \geq 0, x \in\right.$ $\mathbf{R}\}$. Let $\tau(r) \stackrel{\text { def }}{=} \inf \left\{s>0: L_{s}^{0}>r\right\}$ (for $\left.r>0\right)$ denote its inverse local time at 0 . Fix $a>0, b>0, \mu \geq 1$ and $\lambda>0$. We define the function

$$
\mathbf{S}(y) \stackrel{\text { def }}{=} \int_{1 / 2}^{y} \frac{\mathrm{d} x}{x^{a}(1-x)^{b+1}}, \quad y \in(0,1)
$$

and the process

$$
\mathbf{J}(t) \stackrel{\text { def }}{=} \int_{0}^{1} y^{a+\mu-1}(1-y)^{b-\mu-1} L_{\tau(\lambda)}^{\mathbf{S}(y) / t} \mathrm{~d} y, \quad t>0 .
$$

Then we have

$$
\mathbf{J}(t)= \begin{cases}\lambda B(a+\mu, b-\mu)+\delta(t), & b>\mu, \\ c_{5} \lambda+\frac{1}{b}\left(\lambda \log t+\int_{0}^{1} \frac{L_{\tau(\lambda)}^{x}-\lambda}{x} \mathrm{~d} x+\int_{1}^{\infty} \frac{L_{\tau(\lambda)}^{x}}{x} \mathrm{~d} x\right)+\varepsilon(t), & b=\mu, \\ b^{\mu / b-2}\left(\int_{0}^{\infty} x^{\mu / b-2} L_{\tau(\lambda)}^{x} \mathrm{~d} x+\varepsilon(t)\right) t^{\mu / b-1}, & b<\mu,\end{cases}
$$

where $\delta(t) \stackrel{\text { a.s. }}{\longrightarrow} 0($ as $t \rightarrow \infty), B(\cdot, \cdot)$ is the usual beta function, and

$$
c_{5} \stackrel{\text { def }}{=} \frac{\log b}{b}+(a+b-1) \int_{0}^{1} y^{a+b-2} \log (1-y) \mathrm{d} y .
$$

Proof of Lemma 2.3. Looking at the integral expression in $\mathbf{J}(t)$, it is seen that the only possible explosion comes from the neighbourhood of 1 . However, if $b>\mu$, the integral $\int_{0}^{1} y^{a+\mu-1}(1-y)^{b-\mu-1} \mathrm{~d} y$ being finite, Lemma 2.3 follows trivially from the dominated convergence theorem.

It remains to treat the case $b \leq \mu$. Write

$$
\begin{aligned}
\mathbf{J}(t) & =\left(\int_{0}^{1 / 2}+\int_{1 / 2}^{1}\right) y^{a+\mu-1}(1-y)^{b-\mu-1} L_{\tau(\lambda)}^{\mathbf{S}(y) / t} \mathrm{~d} y \\
& \stackrel{\text { def }}{=} \mathbf{J}_{1}(t)+\mathbf{J}_{2}(t) .
\end{aligned}
$$

Again, we can apply the dominated convergence theorem to $\mathbf{J}_{1}(t)$ to arrive at

$$
\mathbf{J}_{1}(t)=\lambda \int_{0}^{1 / 2} y^{a+\mu-1}(1-y)^{b-\mu-1} \mathrm{~d} y+\varepsilon(t) .
$$

We have to deal with $\mathbf{J}_{2}(t)$. Writing $\mathbf{S}^{-1}$ for the inverse function of $\mathbf{S}$, and by a change of variables $x=\mathbf{S}(y) / t$,

$$
\mathbf{J}_{2}(t)=t \int_{0}^{\infty}\left(\mathbf{S}^{-1}(t x)\right)^{2 a+\mu-1}\left(1-\mathbf{S}^{-1}(t x)\right)^{2 b-\mu} L_{\tau(\lambda)}^{x} \mathrm{~d} x .
$$


Observe that

$$
1-\mathbf{S}^{-1}(v) \sim(b v)^{-1 / b}, \quad v \rightarrow \infty .
$$

Therefore, in case $b<\mu$, we have, by the dominated convergence theorem,

$$
\begin{aligned}
\mathbf{J}_{2}(t) & =t^{\mu / b-1} b^{\mu / b-2} \int_{0}^{\infty}\left(\mathbf{S}^{-1}(t x)\right)^{2 a+\mu-1} \frac{\left(1-\mathbf{S}^{-1}(t x)\right)^{2 b-\mu}}{(b t)^{\mu / b-2}} L_{\tau(\lambda)}^{x} \mathrm{~d} x \\
& =t^{\mu / b-1} b^{\mu / b-2}\left(\int_{0}^{\infty} \frac{L_{\tau(\lambda)}^{x}}{x^{2-\mu / b}} \mathrm{~d} x+\varepsilon(t)\right),
\end{aligned}
$$

which, in view of (2.7)-(2.8), yields Lemma 2.3 (the constant term in $\mathbf{J}_{1}(t)$ being absorbed by $\varepsilon(t))$.

Finally, let us assume $b=\mu$. By (2.9),

$$
\begin{aligned}
\mathbf{J}_{2}(t)=t & \int_{1}^{\infty}\left(\mathbf{S}^{-1}(t x)\right)^{2 a+b-1}\left(1-\mathbf{S}^{-1}(t x)\right)^{b} L_{\tau(\lambda)}^{x} \mathrm{~d} x \\
& +t \int_{0}^{1}\left(\mathbf{S}^{-1}(t x)\right)^{2 a+b-1}\left(1-\mathbf{S}^{-1}(t x)\right)^{b}\left(L_{\tau(\lambda)}^{x}-\lambda\right) \mathrm{d} x \\
& +\lambda t \int_{0}^{1}\left(\mathbf{S}^{-1}(t x)\right)^{2 a+b-1}\left(1-\mathbf{S}^{-1}(t x)\right)^{b} \mathrm{~d} x .
\end{aligned}
$$

Now, on the right hand side, we apply the dominated convergence theorem (via observation (2.10), of course) to the first two terms, and use a change of variables $x=\mathbf{S}(y) / t$ for the third term. Accordingly,

$$
\begin{aligned}
\mathbf{J}_{2}(t)=\frac{1}{b} & \int_{1}^{\infty} \frac{L_{\tau(\lambda)}^{x}}{x} \mathrm{~d} x+\frac{1}{b} \int_{0}^{1} \frac{L_{\tau(\lambda)}^{x}-\lambda}{x} \mathrm{~d} x+\varepsilon(t) \\
& +\lambda \int_{1 / 2}^{\mathbf{S}^{-1}(t)} \frac{y^{a+b-1}}{1-y} \mathrm{~d} y .
\end{aligned}
$$

To treat the last integral term on the right hand side, let us recall (2.10) and use integration by parts, which leads to:

$$
\begin{aligned}
\lambda \int_{1 / 2}^{\mathbf{S}^{-1}(t)} & \frac{y^{a+b-1}}{1-y} \mathrm{~d} y=\frac{\lambda \log t}{b}+o(1) \\
& +\lambda\left(\frac{\log b}{b}-\frac{\log 2}{2^{a+b-1}}+(a+b-1) \int_{1 / 2}^{1} y^{a+b-2} \log (1-y) \mathrm{d} y\right) .
\end{aligned}
$$

Combining (2.7)-(2.8) and (2.11)-(2.12) completes the proof of Lemma 2.3.

To get our second preliminary estimate, we have to recall the definition of the Jacobi processes (cf., for example, Karlin and Taylor [18]):

$$
\left\{\begin{aligned}
\mathrm{d} Y(t) & =2 \sqrt{Y(t)(1-Y(t))} \mathrm{d} B(t)+\left(d_{1}-\left(d_{1}+d_{2}\right) Y(t)\right) \mathrm{d} t, \\
Y(0) & =a \in(0,1)
\end{aligned}\right.
$$

where $B$ is a standard Brownian motion. In the literature, $\{Y(t) ; t \geq 0\}$ is sometimes referred to as a Jacobi process of dimensions $\left(d_{1}, d_{2}\right)$, starting from $a$.

We need the following known results for Jacobi processes and Brownian local times. Fact 2.4 is due to Warren and Yor [33]. Fact 2.5 is a consequence of the ergodic theorem on path space, cf. for example Revuz and Yor [26, p. 411]. Fact 2.7 is due to McKean [22] and Ray [24]; see, e.g. Itô and McKean [17, p. 65 et seq.]; this estimate has been extended by Barlow and Marcus-Rosen to a large class of 
Lévy processes. Fact 2.6, which clearly shows where the stable laws in Theorem 1.1 come from, can be found in Biane and Yor [3], except for (2.18), whose proof is postponed until Section 4. We point out that the value of $c_{0}$ in (2.16) is given in (1.5); this corrects a misprint made in Biane and Yor [3, p. 66], in passing from the formula on their (correct) 4th line to their (wrong) 8th line.

Fact 2.4 (Warren and Yor). Let $\left\{R_{1}(t) ; t \geq 0\right\}$ and $\left\{R_{2}(t) ; t \geq 0\right\}$ be independent Bessel processes of dimensions $d_{1}$ and $d_{2}$ respectively, with $d_{1}+d_{2} \geq 2, R_{1}(0)=r_{1} \geq$ 0 and $R_{2}(0)=r_{2}>0$. There exists a Jacobi process $\{Y(t) ; t \geq 0\}$ of dimensions $\left(d_{1}, d_{2}\right)$, starting from $r_{1} / \sqrt{r_{1}^{2}+r_{2}^{2}}$, independent of $\left\{R_{1}^{2}(t)+R_{2}^{2}(t) ; t \geq 0\right\}$, such that for all $t \geq 0$,

$$
\frac{R_{1}^{2}(t)}{R_{1}^{2}(t)+R_{2}^{2}(t)}=Y\left(\int_{0}^{t} \frac{\mathrm{d} s}{R_{1}^{2}(s)+R_{2}^{2}(s)}\right) .
$$

Fact 2.5 (Revuz and Yor). If $\{R(t) ; t \geq 0\}$ is a Bessel process of dimension $d>2$, starting from $r>0$, then

$$
\frac{1}{\log t} \int_{0}^{t} \frac{\mathrm{d} u}{R^{2}(u)} \stackrel{\text { a.s. }}{\longrightarrow} \frac{1}{d-2}, \quad t \rightarrow \infty .
$$

Moreover,

$$
\frac{1}{(\log t)^{1 / 2}}\left(\int_{0}^{t} \frac{\mathrm{d} u}{R^{2}(u)}-\frac{\log t}{d-2}\right) \stackrel{\text { law }}{\longrightarrow} \frac{2}{(d-2)^{3 / 2}} \mathcal{N}, \quad t \rightarrow \infty .
$$

Fact 2.6 (Biane and Yor). In the notation of Lemma 2.3, for any $\lambda>0,0<p<1$ and $1<\alpha<2$,

$$
\begin{gathered}
\int_{0}^{1} \frac{L_{\tau(\lambda)}^{x}-\lambda}{x} \mathrm{~d} x+\int_{1}^{\infty} \frac{L_{\tau(\lambda)}^{x}}{x} \mathrm{~d} x \stackrel{\text { law }}{=} c_{0} \lambda+\frac{\pi}{2} \mathcal{C}_{\lambda}^{\mathrm{ca}}, \\
\int_{0}^{\infty} x^{1 / p-2} L_{\tau(\lambda)}^{x} \mathrm{~d} x \stackrel{\text { law }}{=} 2 p^{2-2 / p} \psi(p) \lambda^{1 / p} \mathcal{S}_{p}^{\mathrm{ca}} \\
\int_{0}^{\tau(\lambda)} \beta^{1 / \alpha-1}(s) \mathbf{1}_{\{\beta(s)>0\}} \mathrm{d} \beta(s) \stackrel{\text { law }}{=} \alpha^{1-2 / \alpha}(\alpha-1) \psi(\alpha) \lambda^{1 / \alpha} \mathcal{S}_{\alpha}^{\mathrm{ca}},
\end{gathered}
$$

where " $=$ law" denotes identity in law.

Fact 2.7 (McKean, Ray). In the notation of Lemma 2.3, for any $T>0$, there exists a (finite) random variable $\Theta(T)$, depending only on $T$, such that

$$
\sup _{0 \leq s \leq T}\left|L_{s}^{x}-L_{s}^{y}\right| \leq \Theta(T) \sqrt{|x-y| \log \frac{1}{|x-y|}},
$$

for all $(x, y) \in \mathbf{R}^{2}$ with $|x-y| \leq 1 / 2$.

Our second preliminary estimate concerns a class of processes which includes $\Xi(t)$ (cf. (2.1)) as a special case.

Lemma 2.8. Let $R_{1}$ and $R_{2}$ be independent Bessel processes of dimensions $d_{1}>0$ and $d_{2}>2$ respectively, with $R_{1}(0)=r_{1} \geq 0$ and $R_{2}(0)=r_{2}>0$. Fix $\mu \geq 1$ and

$$
\Xi_{\mu}(t) \stackrel{\text { def }}{=} \int_{0}^{t} \frac{R_{1}^{2 \mu}(s)}{R_{2}^{2 \mu+2}(s)} \mathrm{d} s, \quad t>0 .
$$


We have, as $t$ goes to infinity,

$$
\begin{aligned}
& \frac{\Xi_{\mu}(t)}{\log t} \stackrel{\text { a.s. }}{\longrightarrow} \frac{B\left(d_{1}^{\prime}+\mu, d_{2}^{\prime}-\mu-1\right)}{\left(d_{1}+d_{2}-2\right) B\left(d_{1}^{\prime}, d_{2}^{\prime}\right)}, \quad d_{2}^{\prime}>\mu+1, \\
& \frac{\Xi_{\mu}(t)-c_{6}(\log t)\left(\log _{2} t\right)}{\log t} \stackrel{\text { law }}{\longrightarrow} c_{7}+c_{8} \mathcal{C}_{4 / B\left(d_{1}^{\prime}, d_{2}^{\prime}\right)}^{\text {ca }}, \quad d_{2}^{\prime}=\mu+1, \\
& \frac{\Xi_{\mu}(t)}{(\log t)^{\mu /\left(d_{2}^{\prime}-1\right)}} \stackrel{\text { law }}{\longrightarrow} c_{9} \mathcal{S}_{\left(d_{2}^{\prime}-1\right) / \mu}^{\mathrm{ca}}, \quad 1<d_{2}^{\prime}<\mu+1,
\end{aligned}
$$

where

$$
\begin{aligned}
& c_{6} \stackrel{\text { def }}{=} \frac{2}{\left(d_{2}-2\right)^{2}} \frac{1}{B\left(d_{1}^{\prime}, d_{2}^{\prime}-1\right)}, \\
& c_{7} \stackrel{\text { def }}{=} \frac{1}{\left(d_{1}+d_{2}-2\right) B\left(d_{1}^{\prime}, d_{2}^{\prime}\right)}\left[\frac{c_{0}+\log \left(\left(d_{2}^{\prime}-1\right) /\left(d_{1}+d_{2}-2\right)\right)}{d_{2}^{\prime}-1}\right. \\
& \\
& \left.\quad+\left(d_{1}^{\prime}+d_{2}^{\prime}-2\right) \int_{0}^{1} y^{d_{1}^{\prime}+d_{2}^{\prime}-3} \log (1-y) \mathrm{d} y\right], \\
& c_{8} \stackrel{\text { def }}{=} \frac{\pi}{4\left(d_{2}-2\right)\left(d_{1}+d_{2}-2\right)}, \\
& c_{9} \stackrel{\text { def }}{=} \frac{1}{2 \mu^{2}} \psi\left(\frac{d_{2}^{\prime}-1}{\mu}\right)\left[\frac{2 \mu^{2}}{\left(d_{2}^{\prime}-1\right)^{2} B\left(d_{1}^{\prime}, d_{2}^{\prime}-1\right)}\right]^{\mu /\left(d_{2}^{\prime}-1\right)} .
\end{aligned}
$$

Proof. By (2.13), there exists a Jacobi process $\{Y(t) ; t \geq 0\}$ of dimensions $\left(d_{1}, d_{2}\right)$, such that

$$
\Xi_{\mu}(t)=\int_{0}^{\Lambda(t)} \frac{Y^{\mu}(s)}{(1-Y(s))^{\mu+1}} \mathrm{~d} s=\Upsilon_{\mu}(\Lambda(t)),
$$

where

$$
\begin{gathered}
\Upsilon_{\mu}(v) \stackrel{\text { def }}{=} \int_{0}^{v} \frac{Y^{\mu}(s)}{(1-Y(s))^{\mu+1}} \mathrm{~d} s, \\
\Lambda(t) \stackrel{\text { def }}{=} \int_{0}^{t} \frac{\mathrm{d} u}{R_{1}^{2}(u)+R_{2}^{2}(u)} .
\end{gathered}
$$

Since $R_{1}^{2}+R_{2}^{2}$ is a squared Bessel process of dimension $\left(d_{1}+d_{2}\right)$, Fact 2.5 confirms that, as $t$ tends to infinity,

$$
\begin{aligned}
& \frac{\Lambda(t)}{\log t} \stackrel{\text { a.s. }}{\longrightarrow} \frac{1}{d_{1}+d_{2}-2}, \\
& \frac{1}{(\log t)^{1 / 2}}\left(\Lambda(t)-\frac{\log t}{d_{1}+d_{2}-2}\right) \stackrel{\text { law }}{\longrightarrow} \frac{2}{\left(d_{1}+d_{2}-2\right)^{3 / 2}} \mathcal{N} .
\end{aligned}
$$

We now study the asymptotic behaviour of the process $\Upsilon_{\mu}(v)$ (cf. (2.23)). By the strong Markov property, we can assume without loss of generality that $Y(0)=1 / 2$. A scale function of $Y$ is

$$
S(y) \stackrel{\text { def }}{=} \int_{1 / 2}^{y} \frac{\mathrm{d} x}{x^{d_{1}^{\prime}}(1-x)^{d_{2}^{\prime}}}, \quad y \in(0,1) .
$$

Hence, $t \mapsto S(Y(t))$ is a continuous local martingale, and there exists a Brownian motion $\beta$, with $\beta(0)=0$, such that for all $t \geq 0$,

$$
S(Y(t))=\beta(U(t)),
$$


where

$$
U(t) \stackrel{\text { def }}{=} 4 \int_{0}^{t} \frac{\mathrm{d} s}{Y^{d_{1}-1}(s)(1-Y(s))^{d_{2}-1}},
$$

with $U(\infty)=\inf \{t>0: \beta(t)=S(0)\}$ (hence $U(\infty)=\infty$ if $S(0)=-\infty$ ). Consider the inverse process of $U$ :

$$
\begin{aligned}
U^{-1}(t) & \stackrel{\text { def }}{=} \inf \{s>0: U(s)>t\} \\
& =\frac{1}{4} \int_{0}^{t}\left(S^{-1}(\beta(s))\right)^{d_{1}-1}\left(1-S^{-1}(\beta(s))\right)^{d_{2}-1} \mathrm{~d} s,
\end{aligned}
$$

where $S^{-1}$ is the inverse of $S$. Write $\left\{L_{t}^{x}(\beta) ; t \geq 0, x \in \mathbf{R}\right\}$ for the bicontinuous local time process of $\beta$. Applying the occupation density formula gives

$$
U^{-1}(t)=\frac{1}{4} \int_{0}^{1} x^{d_{1}^{\prime}-1}(1-x)^{d_{2}^{\prime}-1} L_{t}^{S(x)}(\beta) \mathrm{d} x .
$$

Recall $\Upsilon_{\mu}(v)$ from (2.23). By (2.29),

$$
\begin{aligned}
\Upsilon_{\mu}(v) & =\int_{0}^{v} \frac{Y^{\mu}(s)}{(1-Y(s))^{\mu+1}} \frac{1}{4} Y^{d_{1}-1}(s)(1-Y(s))^{d_{2}-1} \mathrm{~d} U(s) \\
& =\frac{1}{4} \int_{0}^{v} Y^{d_{1}+\mu-1}(s)(1-Y(s))^{d_{2}-\mu-2} \mathrm{~d} U(s) \\
& =\frac{1}{4} \int_{0}^{U(v)}\left(S^{-1}(\beta(u))\right)^{d_{1}+\mu-1}\left(1-S^{-1}(\beta(u))\right)^{d_{2}-\mu-2} \mathrm{~d} u,
\end{aligned}
$$

where in the last equality we used the representation (2.28). By the occupation density formula,

$$
\begin{aligned}
\Upsilon_{\mu}(v) & =\frac{1}{4} \int_{S(0)}^{\infty}\left(S^{-1}(y)\right)^{d_{1}+\mu-1}\left(1-S^{-1}(y)\right)^{d_{2}-\mu-2} L_{U(v)}^{y}(\beta) \mathrm{d} y \\
& =\frac{1}{4} \int_{0}^{1} x^{d_{1}^{\prime}+\mu-1}(1-x)^{d_{2}^{\prime}-\mu-2} L_{U(v)}^{S(x)}(\beta) \mathrm{d} x .
\end{aligned}
$$

Now, for each fixed $v>0$, we introduce the Brownian motion:

$$
\beta_{v}(s) \stackrel{\text { def }}{=} \frac{1}{v} \beta\left(v^{2} s\right), \quad s \geq 0 .
$$

Denote its local time process by $L\left(\beta_{v}\right)$, and its inverse local time at 0 by $\tau_{\beta_{v}}(\cdot)$. According to (2.30),

$$
\begin{aligned}
\frac{1}{v} U^{-1}\left(v^{2} s\right) & =\frac{1}{4} \int_{0}^{1} x^{d_{1}^{\prime}-1}(1-x)^{d_{2}^{\prime}-1} L_{s}^{S(x) / v}\left(\beta_{v}\right) \mathrm{d} x \\
& =\frac{1}{4} B\left(d_{1}^{\prime}, d_{2}^{\prime}\right) L_{s}^{0}\left(\beta_{v}\right)+\varepsilon_{1},
\end{aligned}
$$

where $B(\cdot, \cdot)$ is as before the beta function, and

$$
\varepsilon_{1}=\varepsilon_{1}(v, s) \stackrel{\text { def }}{=} \frac{1}{4} \int_{0}^{1} x^{d_{1}^{\prime}-1}(1-x)^{d_{2}^{\prime}-1}\left(L_{s}^{S(x) / v}\left(\beta_{v}\right)-L_{s}^{0}\left(\beta_{v}\right)\right) \mathrm{d} x .
$$

Writing, for brevity,

$$
\lambda \stackrel{\text { def }}{=} \frac{4}{B\left(d_{1}^{\prime}, d_{2}^{\prime}\right)},
$$


it is easily seen using Fact 2.7 that $\sup _{0 \leq s \leq \tau_{\beta}(2 \lambda)}\left|\varepsilon_{1}\right| \stackrel{\mathrm{P} .}{\longrightarrow} 0$, as $v$ tends to infinity. Let $\delta>0$ be a small number. On the event $\left\{\sup _{0 \leq s \leq \tau_{\beta_{v}}(2 \lambda)}\left|\varepsilon_{1}\right|<\delta\right\}$,

$$
\tau_{\beta_{v}}((1-\delta) \lambda) \leq \frac{U(v)}{v^{2}} \leq \tau_{\beta_{v}}((1+\delta) \lambda) .
$$

Going back to (2.31), we have, on $\left\{\sup _{0 \leq s \leq \tau_{\beta_{v}}(2 \lambda)}\left|\varepsilon_{1}\right|<\delta\right\}$,

$$
\begin{aligned}
\frac{v}{4} \int_{0}^{1} x^{d_{1}^{\prime}+\mu-1}(1-x)^{d_{2}^{\prime}-\mu-2} L_{\tau_{\beta_{v}}((1-\delta) \lambda)}^{S(x)}\left(\beta_{v}\right) \mathrm{d} x \leq \Upsilon_{\mu}(v) \\
\leq \frac{v}{4} \int_{0}^{1} x^{d_{1}^{\prime}+\mu-1}(1-x)^{d_{2}^{\prime}-\mu-2} L_{\tau_{\beta_{v}}((1+\delta) \lambda)}^{S(x) / v}\left(\beta_{v}\right) \mathrm{d} x .
\end{aligned}
$$

We now complete the proof of Lemma 2.8 by discussing three possible situations.

(i) In case $d_{2}^{\prime}>\mu+1$, this is an immediate consequence of the ergodic theorem on path space.

(ii) Assume $d_{2}^{\prime}=\mu+1$. In view of (2.35), applying Lemma 2.3 to $a=d_{1}^{\prime}$ and $b=d_{2}^{\prime}-1$ gives (recalling $\lambda$ from (2.33))

$$
\begin{aligned}
\Upsilon_{\mu}(v)= & \frac{\lambda v}{4}\left(\frac{\log \left(d_{2}^{\prime}-1\right)}{d_{2}^{\prime}-1}+\left(d_{1}^{\prime}+d_{2}^{\prime}-2\right) \int_{0}^{1} y^{d_{1}^{\prime}+d_{2}^{\prime}-3} \log (1-y) \mathrm{d} y\right) \\
& +\frac{v}{2\left(d_{2}-2\right)}\left(\lambda \log v+\int_{0}^{1} \frac{L_{\tau(\lambda)}^{x}-\lambda}{x} \mathrm{~d} x+\int_{1}^{\infty} \frac{L_{\tau(\lambda)}^{x}}{x} \mathrm{~d} x\right)+v \varepsilon(v) .
\end{aligned}
$$

In light of (2.16), combining (2.36) with (2.26) (we do need (2.26) here, instead of (2.25) only, to ensure that the error term in (2.25) is negligible) and (2.22) yields (2.20).

(iii) Finally, let us treat the case $d_{2}^{\prime}<\mu+1$. Again, we can use (2.35) and Lemma 2.3 to see that

$$
\frac{\Upsilon_{\mu}(v)}{v^{\mu /\left(d_{2}^{\prime}-1\right)}} \stackrel{\text { law }}{\longrightarrow} \frac{1}{4}\left(d_{2}^{\prime}-1\right)^{\mu /\left(d_{2}^{\prime}-1\right)-2} \int_{0}^{\infty} x^{\mu /\left(d_{2}^{\prime}-1\right)-2} L_{\tau(\lambda)}^{x} \mathrm{~d} x
$$

Applying (2.17) to $p=\left(d_{2}^{\prime}-1\right) / \mu$ and using (2.25) and (2.22), we readily get $(2.21)$.

Proof of Proposition 2.1. The estimates (2.2)-(2.3) being already proved in Lemma 2.8 (taking $\mu=1$ there); we only have to check (2.4)-(2.6). So let us assume $d_{2}>4$.

We write the stochastic differential equations satisfied by $R_{1}$ and $R_{2}$ :

$$
\begin{aligned}
& R_{1}^{2}(t)=r_{1}^{2}+2 \int_{0}^{t} R_{1}(s) \mathrm{d} \beta_{1}(s)+d_{1} t \\
& R_{2}^{2}(t)=r_{2}^{2}+2 \int_{0}^{t} R_{2}(s) \mathrm{d} \beta_{2}(s)+d_{2} t
\end{aligned}
$$

where $\beta_{1}$ and $\beta_{2}$ are independent real-valued Brownian motions, both starting from 0 . Applying Itô's formula to $R_{1}^{2}(t) / R_{2}^{2}(t)$ gives

$$
\begin{aligned}
\left(d_{2}-4\right) \Xi(t)=d_{1} \int_{0}^{t} & \frac{\mathrm{d} s}{R_{2}^{2}(s)}+\frac{r_{1}^{2}}{r_{2}^{2}}-\frac{R_{1}^{2}(t)}{R_{2}^{2}(t)} \\
& +2 \int_{0}^{t}\left(\frac{R_{1}(s) \mathrm{d} \beta_{1}(s)}{R_{2}^{2}(s)}-\frac{R_{1}^{2}(s) \mathrm{d} \beta_{2}(s)}{R_{2}^{3}(s)}\right) .
\end{aligned}
$$


On the other hand, observe that

$$
\log R_{2}(t)-\log r_{2}=\int_{0}^{t} \frac{\mathrm{d} \beta_{2}(s)}{R_{2}(s)}+\left(d_{2}^{\prime}-1\right) \int_{0}^{t} \frac{\mathrm{d} s}{R_{2}^{2}(s)}
$$

which, in view of (2.39), implies

$$
\begin{aligned}
Z(t) & \stackrel{\text { def }}{=} \frac{\left(d_{2}-4\right)\left(d_{2}^{\prime}-1\right)}{d_{1}} \Xi(t)-\log R_{2}(t)+\log r_{2}-\frac{d_{2}^{\prime}-1}{d_{1}}\left(\frac{r_{1}^{2}}{r_{2}^{2}}-\frac{R_{1}^{2}(t)}{R_{2}^{2}(t)}\right) \\
& =\frac{d_{2}-2}{d_{1}} \int_{0}^{t} \frac{R_{1}(s) \mathrm{d} \beta_{1}(s)}{R_{2}^{2}(s)}-\int_{0}^{t}\left(\frac{1}{R_{2}(s)}+\frac{\left(d_{2}-2\right) R_{1}^{2}(s)}{d_{1} R_{2}^{3}(s)}\right) \mathrm{d} \beta_{2}(s) \\
& =\gamma(\Delta(t)) .
\end{aligned}
$$

Let us explain the identity (2.43). The expression (2.42) being a continuous local martingale, it follows from the well known Dubins-Schwarz representation theorem that (2.43) holds for some Brownian motion $\gamma$ and for $\Delta(t)$ equal to the increasing process associated with this local martingale, i.e.

$$
\begin{aligned}
& \Delta(t)=\left(\frac{d_{2}-2}{d_{1}}\right)^{2} \int_{0}^{t} \frac{R_{1}^{2}(s)}{R_{2}^{4}(s)} \mathrm{d} s+\int_{0}^{t}\left(\frac{1}{R_{2}(s)}+\frac{\left(d_{2}-2\right) R_{1}^{2}(s)}{d_{1} R_{2}^{3}(s)}\right)^{2} \mathrm{~d} s \\
&= \int_{0}^{t} \frac{\mathrm{d} s}{R_{2}^{2}(s)}+\frac{\left(d_{2}-2\right)\left(d_{2}-2+2 d_{1}\right)}{d_{1}^{2}} \int_{0}^{t} \frac{R_{1}^{2}(s)}{R_{2}^{4}(s)} \mathrm{d} s \\
& \quad+\left(\frac{d_{2}-2}{d_{1}}\right)^{2} \int_{0}^{t} \frac{R_{1}^{4}(s)}{R_{2}^{6}(s)} \mathrm{d} s \\
& \stackrel{\text { def }}{=} \Delta_{1}(t)+\Delta_{2}(t)+\Delta_{3}(t) .
\end{aligned}
$$

Before estimating $\Delta_{j}(t)$ (for $1 \leq j \leq 3$ ), let us look at $Z(t)$ in (2.41). By the usual law of the iterated logarithm and the Dvoretzky-Erdös [11] integral test for the rate of escape of transient Bessel processes, one can easily check that $R_{1}^{2}(t) / R_{2}^{2}(t)=$ $o\left((\log t)^{1 / 2}\right)$ and that $\log R_{2}(t)=(\log t) / 2+O\left(\log _{2} t\right)$ almost surely. Therefore,

$$
Z(t)=\frac{\left(d_{2}-4\right)\left(d_{2}-2\right)}{2 d_{1}} \Xi(t)-\frac{1}{2} \log t+o\left((\log t)^{1 / 2}\right), \quad \text { a.s. }
$$

Applying (2.43) and (2.44) yields

$$
\frac{\left(d_{2}-4\right)\left(d_{2}-2\right)}{2 d_{1}} \Xi(t)=\frac{1}{2} \log t+\gamma\left(\sum_{j=1}^{3} \Delta_{j}(t)\right)+o\left((\log t)^{1 / 2}\right), \quad \text { a.s. }
$$

Let us estimate the $\Delta_{j}(t)$ 's. By Fact 2.5,

$$
\frac{\Delta_{1}(t)}{\log t} \stackrel{\text { a.s. }}{\longrightarrow} \frac{1}{d_{2}-2} .
$$

Applying (2.19) to $\int_{0}^{t}\left(R_{1}^{2}(s) / R_{2}^{4}(s)\right) \mathrm{d} s$ gives that, as $t$ tends to infinity,

$$
\frac{\Delta_{2}(t)}{\log t} \stackrel{\text { a.s. }}{\longrightarrow} \frac{2 d_{1}+d_{2}-2}{d_{1}\left(d_{2}-4\right)}, \quad \text { a.s. }
$$

We now complete the proof of Proposition 2.1 by verifying (2.6), (2.5) and (2.4) separately. 
Proof of (2.6). Assume $d_{2}>6$. In this case, Lemma 2.8 (with $\mu=2$ ) confirms that

$$
\frac{\Delta_{3}(t)}{\log t} \stackrel{\text { a.s. }}{\longrightarrow} \frac{\left(d_{1}+2\right)\left(d_{2}-2\right)}{d_{1}\left(d_{2}-4\right)\left(d_{2}-6\right)}, \quad \text { a.s. },
$$

which, in view of (2.46)-(2.48), yields (2.6).

Proof of (2.5). Let $d_{2}=6$. By Lemma 2.8 (recalling that $\lambda \stackrel{\text { def }}{=} 4 / B\left(d_{1}^{\prime}, d_{2}^{\prime}\right)$ ),

$$
\frac{\Delta_{3}(t)}{(\log t)\left(\log _{2} t\right)} \stackrel{\text { P. }}{\longrightarrow} \frac{d_{1}^{\prime}+1}{d_{1}} \text {. }
$$

This, jointly considered with (2.46)-(2.48), implies (2.5).

Proof of (2.4). Assume now $4<d_{2}<6$. Let us go back to $(2.42)$ :

$$
Z(t)=\frac{d_{2}-2}{d_{1}} \int_{0}^{t}\left(\frac{R_{1}(s)}{R_{2}^{2}(s)} \mathrm{d} \beta_{1}(s)-\frac{R_{1}^{2}(s)}{R_{2}^{3}(s)} \mathrm{d} \beta_{2}(s)\right)-\int_{0}^{t} \frac{\mathrm{d} \beta_{2}(s)}{R_{2}(s)} .
$$

The last term on the right hand side is easy to handle. Indeed, it is a continuous martingale whose increasing process is $t \mapsto \int_{0}^{t}\left(\mathrm{~d} s / R_{2}^{2}(s)\right)$. By (2.14), $\int_{0}^{t}\left(\mathrm{~d} s / R_{2}^{2}(s)\right) \sim(\log t) /\left(d_{2}-2\right)$ almost surely when $t \rightarrow \infty$. Applying the DubinsSchwarz theorem yields

$$
\frac{1}{(\log t)^{1 / 2}} \int_{0}^{t} \frac{\mathrm{d} \beta_{2}(s)}{R_{2}(s)} \stackrel{\text { law }}{\longrightarrow} \frac{\mathcal{N}}{\left(d_{2}-2\right)^{1 / 2}}, \quad t \rightarrow \infty .
$$

(Indeed, this is an equivalent form of $(2.15))$. Since $1 /\left(d_{2}^{\prime}-1\right)>1 / 2$ and since $\log \left(R_{2}(t) / \sqrt{t}\right)$ converges in law, in view of $(2.45)$, it remains only to show that

$$
\frac{M(t)}{(\log t)^{1 /\left(d_{2}^{\prime}-1\right)}} \stackrel{\text { law }}{\longrightarrow}\left(d_{2}^{\prime}-2\right) c_{2} \mathcal{S}_{d_{2}^{\prime}-1}^{\mathrm{ca}}, \quad t \rightarrow \infty,
$$

where

$$
M(t) \stackrel{\text { def }}{=} \int_{0}^{t}\left(\frac{R_{1}(s)}{R_{2}^{2}(s)} \mathrm{d} \beta_{1}(s)-\frac{R_{1}^{2}(s)}{R_{2}^{3}(s)} \mathrm{d} \beta_{2}(s)\right) .
$$

To this end, we use an argument similar to that in the proof of Lemma 2.8. Indeed, Fact 2.4 guarantees the existence of a Jacobi process $Y$ of dimensions $\left(d_{1}, d_{2}\right)$ with $Y(0)=r_{1} / \sqrt{r_{1}^{2}+r_{2}^{2}}$ satisfying (2.13). Recall $\Lambda(t)$ from (2.24). By the DubinsSchwarz theorem, there exists a Brownian motion $B$ such that

$$
\int_{0}^{t} \frac{R_{2}(s) \mathrm{d} \beta_{1}(s)-R_{1}(s) \mathrm{d} \beta_{2}(s)}{R_{1}^{2}(s)+R_{2}^{2}(s)}=B(\Lambda(t)) .
$$

We point out that, according to Knight's theorem on continuous orthogonal martingales and in view of (2.37)-(2.38), the Brownian motion $B$ is independent of $t \mapsto R_{1}^{2}(t)+R_{2}^{2}(t)$, therefore of $t \mapsto \Lambda(t)$.

It is an immediate consequence of (2.50) that

$$
\begin{aligned}
M(t) & =\int_{0}^{t} \frac{R_{1}(s)\left(R_{1}^{2}(s)+R_{2}^{2}(s)\right)}{R_{2}^{3}(s)} \frac{R_{2}(s) \mathrm{d} \beta_{1}(s)-R_{1}(s) \mathrm{d} \beta_{2}(s)}{R_{1}^{2}(s)+R_{2}^{2}(s)} \\
& =\int_{0}^{\Lambda(t)} \frac{Y^{1 / 2}(u)}{(1-Y(u))^{3 / 2}} \mathrm{~d} B(u),
\end{aligned}
$$


where the last equality follows from (2.13). For notational convenience, write

$$
\begin{aligned}
& N_{+}(v) \stackrel{\text { def }}{=} \int_{0}^{v} \frac{Y^{1 / 2}(u)}{(1-Y(u))^{3 / 2}} \mathbf{1}_{\{Y(u)>1 / 2\}} \mathrm{d} B(u), \\
& N_{-}(v) \stackrel{\text { def }}{=} \int_{0}^{v} \frac{Y^{1 / 2}(u)}{(1-Y(u))^{3 / 2}} \mathbf{1}_{\{Y(u) \leq 1 / 2\}} \mathrm{d} B(u) .
\end{aligned}
$$

(Therefore $M(t)=N_{+}(\Lambda(t))+N_{-}(\Lambda(t))$ for all $t$.) The increasing process associated with the continuous martingale $N_{-}$is $\int_{0}^{v} Y(u)(1-Y(u))^{-3} \mathbf{1}_{\{Y(u) \leq 1 / 2\}} \mathrm{d} u$, which is smaller than $4 v$. It follows from the Dubins-Schwarz theorem and the usual iterated logarithm law for Brownian motion that for all $\varepsilon>0, N_{-}(v) / v^{1 / 2+\varepsilon} \stackrel{\text { a.s. }}{\longrightarrow} 0$ $(v \rightarrow \infty)$. Since $M(t)=N(\Lambda(t))$, with $\Lambda(t) / \log t \stackrel{\text { a.s. }}{\longrightarrow} 1 /\left(d_{1}+d_{2}-2\right)$ (cf. (2.25)), the proof of (2.49) is reduced to showing the following estimate:

$$
\frac{N_{+}(v)}{v^{1 /\left(d_{2}^{\prime}-1\right)}} \stackrel{\text { law }}{\longrightarrow}\left(d_{2}^{\prime}-2\right)\left(d_{1}+d_{2}-2\right)^{1 /\left(d_{2}^{\prime}-1\right)} c_{2} \mathcal{S}_{d_{2}^{\prime}-1}^{\text {ca }}, \quad v \rightarrow \infty .
$$

A key observation is that the Brownian motion $B$ introduced in (2.50) is also the Brownian motion in the stochastic differential equation satisfied by $Y$, i.e.

$$
\mathrm{d} Y(t)=2 \sqrt{Y(t)(1-Y(t))} \mathrm{d} B(t)+\left(d_{1}-\left(d_{1}+d_{2}\right) Y(t)\right) \mathrm{d} t .
$$

(Indeed, this observation yields the skew-product result presented in Fact 2.4.) Recall $S$ from (2.27), which is a scale function of $Y$. We have

$$
\mathrm{d} S(Y(t))=2 Y^{\left(1-d_{1}\right) / 2}(t)(1-Y(t))^{\left(1-d_{2}\right) / 2} \mathrm{~d} B(t) .
$$

Consequently,

$$
\begin{aligned}
& N_{+}(v) \\
& =\int_{0}^{v} \frac{Y^{1 / 2}(u)}{(1-Y(u))^{3 / 2}} \frac{1}{2} Y^{\left(d_{1}-1\right) / 2}(u)(1-Y(u))^{\left(d_{2}-1\right) / 2} \mathbf{1}_{\{Y(u)>1 / 2\}} \mathrm{d} S(Y(u)) .
\end{aligned}
$$

According to (2.28)-(2.29) (for some Brownian motion $\beta$ ), this yields

$$
\begin{aligned}
N_{+}(v) & =\frac{1}{2} \int_{0}^{v} Y^{d_{1}^{\prime}}(u)(1-Y(u))^{d_{2}^{\prime}-2} \mathbf{1}_{\{Y(u)>1 / 2\}} \mathrm{d} \beta(U(u)) \\
& =\frac{1}{2} \int_{0}^{U(v)}\left(S^{-1}(\beta(s))\right)^{d_{1}^{\prime}}\left(1-S^{-1}(\beta(s))\right)^{d_{2}^{\prime}-2} \mathbf{1}_{\{\beta(s)>0\}} \mathrm{d} \beta(s),
\end{aligned}
$$

where $S^{-1}$ is as before the inverse function of $S$. Write, for brevity,

$$
g(x) \stackrel{\text { def }}{=}\left(S^{-1}(x)\right)^{d_{1}^{\prime}}\left(1-S^{-1}(x)\right)^{d_{2}^{\prime}-2} \mathbf{1}_{\{x>0\}}, \quad x \in \mathbf{R},
$$

which is a deterministic function. Define as in $(2.32)$ the Brownian motion $\beta_{v}(\cdot)=$ $\beta\left(v^{2} \cdot\right) / v$, and $\tau_{\beta_{v}}(\cdot)$, its inverse local time at 0 . We have

$$
N_{+}(v)=\frac{1}{2} \int_{0}^{U(v)} g(\beta(s)) \mathrm{d} \beta(s)=\frac{v}{2} \int_{0}^{U(v) / v^{2}} g\left(v \beta_{v}(s)\right) \mathrm{d} \beta_{v}(s) .
$$

Let $\lambda=4 / B\left(d_{1}^{\prime}, d_{2}^{\prime}\right)$ as in (2.33). It is easily checked that (writing $q \stackrel{\text { def }}{=}$ $\left.\left(d_{2}-4\right) /\left(d_{2}-2\right)\right)$

$$
g(x) \sim\left(\left(d_{2}^{\prime}-1\right) x\right)^{-q}, \quad x \rightarrow \infty .
$$


Let $\tau$ be the inverse local time at 0 of $\beta$. It follows from a martingale convergence theorem (cf. Revuz and Yor [26, Exercise IV.4.14]) that, as $v$ tends to infinity,

$$
v^{q} \int_{0}^{\tau(\lambda)} g(v \beta(s)) \mathrm{d} \beta(s) \stackrel{\mathrm{P} .}{\longrightarrow}\left(d_{2}^{\prime}-1\right)^{-q} \int_{0}^{\tau(\lambda)} \beta^{-q}(s) \mathbf{1}_{\{\beta(s)>0\}} \mathrm{d} \beta(s) .
$$

Note from (2.18) that the law of the last variable (recalling that $\lambda=4 / B\left(d_{1}^{\prime}, d_{2}^{\prime}\right)$ ) is $c_{10} \mathcal{S}_{d_{2}^{\prime}-1}^{\mathrm{ca}}$, where

Since

$$
c_{10} \stackrel{\text { def }}{=}\left(d_{2}^{\prime}-2\right)\left(\frac{4}{\left(d_{2}^{\prime}-1\right) B\left(d_{1}^{\prime}, d_{2}^{\prime}\right)}\right)^{1 /\left(d_{2}^{\prime}-1\right)} \psi\left(d_{2}^{\prime}-1\right) .
$$

we obtain

$$
\int_{0}^{\tau_{\beta_{v}}(\lambda)} g\left(v \beta_{v}(s)\right) \mathrm{d} \beta_{v}(s) \stackrel{\text { law }}{=} \int_{0}^{\tau(\lambda)} g(v \beta(s)) \mathrm{d} \beta(s)
$$

$$
v^{q} \int_{0}^{\tau_{\beta_{v}}(\lambda)} g\left(v \beta_{v}(s)\right) \mathrm{d} \beta_{v}(s) \stackrel{\text { law }}{\longrightarrow} c_{10} \mathcal{S}_{d_{2}^{\prime}-1}^{\mathrm{ca}}, \quad v \rightarrow \infty .
$$

In view of (2.34), we have actually proved the following convergence:

$$
v^{q} \int_{0}^{U(v) / v^{2}} g\left(v \beta_{v}(s)\right) \mathrm{d} \beta_{v}(s) \stackrel{\text { law }}{\longrightarrow} c_{10} \mathcal{S}_{d_{2}^{\prime}-1}^{\mathrm{ca}}, \quad v \rightarrow \infty .
$$

Going back to $(2.52)$ gives

$$
\frac{N_{+}(v)}{v^{1 /\left(d_{2}^{\prime}-1\right)}} \stackrel{\text { law }}{\longrightarrow} \frac{c_{10}}{2} \mathcal{S}_{d_{2}^{\prime}-1}^{\mathrm{ca}}, \quad v \rightarrow \infty .
$$

This yields (2.51), and therefore completes the proof of (2.4).

\section{Proof of Theorem 1.1}

Since (1.13) is proved by Kawazu and Tanaka [20] (cf. Remark 1.2), we shall only check (1.10)-(1.12), and also obtain (1.7)-(1.8) as a by-product.

Let $\{X(t) ; t \geq 0\}$ be a diffusion process with drifted Brownian potential as in (1.2). Let

$$
A(x) \stackrel{\text { def }}{=} \int_{0}^{x} \mathrm{e}^{V(y)} \mathrm{d} y, \quad x \in \mathbf{R}
$$

which is a scale function of $X$. Note that $A(\infty)<\infty$ almost surely, since $\kappa>$ 0 . Using diffusion (or martingale) theory, one can construct a Brownian motion $\{B(t) ; t \geq 0\}$ starting from 0 , independent of the drifted Brownian potential $V$, such that

$$
A(X(t))=B(E(t))
$$

where $E(t) \stackrel{\text { def }}{=}\langle A \circ X\rangle(t)$ denotes the increasing process associated with the local martingale $A \circ X$. Note that the representation (3.2) involves only $\{B(u) ; u<$ $E(\infty)\}$. Define the first hitting times of $B$ :

$$
\sigma(r) \stackrel{\text { def }}{=} \inf \{t>0: B(t)>r\}, \quad r>0 .
$$

Then $E(\infty)=\sigma(A(\infty))$. Now, let

$$
T(r) \stackrel{\text { def }}{=} E^{-1}(r)=\int_{0}^{r} \exp \left(-2 V\left(A^{-1}(B(s))\right)\right) \mathrm{d} s, \quad 0 \leq r<E(\infty),
$$


and we get the following representation for $X$ (cf. Brox [5]):

$$
X(t)=A^{-1}\left(B\left(T^{-1}(t)\right)\right), \quad t \geq 0 .
$$

Let $H$ be as in (1.4), denoting the first hitting time process associated with $X$. Let $\left\{L_{t}^{x}(B) ; t \geq 0, x \in \mathbf{R}\right\}$ be the local time of $B$. By (3.3), for any $r>0$,

$$
\begin{aligned}
H(r) & =T(\sigma(A(r))) \\
& =\int_{0}^{\sigma(A(r))} \exp \left(-2 V\left(A^{-1}(B(s))\right)\right) \mathrm{d} s \\
& =\int_{-\infty}^{A(r)} \mathrm{e}^{-2 V\left(A^{-1}(x)\right)} L_{\sigma(A(r))}^{x}(B) \mathrm{d} x \\
& =I_{1}(r)+I_{2}(r),
\end{aligned}
$$

where

$$
\begin{aligned}
& I_{1}(r) \stackrel{\text { def }}{=} \int_{-\infty}^{0} \mathrm{e}^{-2 V\left(A^{-1}(x)\right)} L_{\sigma(A(r))}^{x}(B) \mathrm{d} x, \\
& I_{2}(r) \stackrel{\text { def }}{=} \int_{0}^{A(r)} \mathrm{e}^{-2 V\left(A^{-1}(x)\right)} L_{\sigma(A(r))}^{x}(B) \mathrm{d} x .
\end{aligned}
$$

We treat the terms $I_{1}(r)$ and $I_{2}(r)$ separately. For the former, let us use a change of variables $x=A(y)$ to arrive at

$$
I_{1}(r)=\int_{-\infty}^{0} \mathrm{e}^{-V(y)} L_{\sigma(A(r))}^{A(y)}(B) \mathrm{d} y .
$$

Recall that $A(\infty)<\infty$ and that $\int_{-\infty}^{0} \mathrm{e}^{-V(y)} \mathrm{d} y<\infty$ almost surely. Therefore,

$$
\sup _{r \geq 0} I_{1}(r)=I_{1}(\infty)<\infty, \quad \text { a.s. }
$$

It remains to handle the second term $I_{2}(r)$. Recall that $V$ is a Brownian motion with negative drift, cf. (1.1). According to the well known (Lamperti) relation between drifted Brownian motion and Bessel processes (cf. Yor [34]), there exists a $\operatorname{BES}(2-2 \kappa)$, denoted by $\{R(t) ; t \geq 0\}$, with $R(0)=2$, such that

$$
\mathrm{e}^{V(t) / 2}=\frac{1}{2} R(A(t)), \quad t \geq 0
$$

where $A$ is as in (3.1). Moreover, $A(\infty)(<\infty$, almost surely) is the first hitting time of $R$ at 0 (i.e. the life time of $R$ ). Define

$$
\Re(t) \stackrel{\text { def }}{=} R(A(\infty)-t), \quad 0 \leq t \leq A(\infty) .
$$

By Williams' time reversal theorem for Bessel processes (cf. for example Revuz and Yor [26, Exercise XI.1.23]), $\Re$ is a (transient) $\operatorname{BES}(2+2 \kappa)$, starting from 0 , such that $A(\infty)$ is the last exit time of $\Re$ from 2 . Accordingly, (3.8) becomes $\exp \left(V\left(A^{-1}(t)\right) / 2\right)=\Re(A(\infty)-t) / 2$ for all $0<t<A(\infty)$. Going back to (3.6), this yields

$$
I_{2}(r)=16 \int_{A(\infty)-A(r)}^{A(\infty)} \frac{L_{\sigma(A(r))}^{A(\infty)-s}(B)}{\Re^{4}(s)} \mathrm{d} s .
$$


According to the first Ray-Knight theorem, $\left\{\frac{1}{A(r)} L_{\sigma(A(r))}^{(1-t) A(r)}(B) ; 0 \leq t \leq 1\right\}$ is a $\operatorname{BESQ}(2)$ starting from 0 , independent of $V$. Therefore, letting $R_{1}$ denote a $\operatorname{BES}(2)$ with $R_{1}(0)=0$,

$$
\begin{aligned}
I_{2}(r) & \stackrel{\text { law }}{=} 16 \int_{A(\infty)-A(r)}^{A(\infty)} \frac{R_{1}^{2}(A(r)+s-A(\infty))}{\Re^{4}(s)} \mathrm{d} s \\
& =16 \int_{0}^{A(r)} \frac{R_{1}^{2}(v)}{\Re^{4}(v+A(\infty)-A(r))} \mathrm{d} v,
\end{aligned}
$$

by a change of variables $v=s+A(r)-A(\infty)$. Write $D(r) \stackrel{\text { def }}{=} A(\infty)-A(r)=$ $\int_{r}^{\infty} \mathrm{e}^{V(y)} \mathrm{d} y$. With the aide of the usual law of the iterated logarithm, it is easily checked that

$$
\log D(r)=-\frac{\kappa}{2} r+O\left(\left(r \log _{2} r\right)^{1 / 2}\right), \quad \text { a.s. }
$$

Going back to (3.9), we have

$$
\begin{aligned}
I_{2}(r) & =16 \int_{0}^{A(\infty)-D(r)} \frac{R_{1}^{2}(v)}{\Re^{4}(v+D(r))} \mathrm{d} v \\
& =16\left(\int_{1}^{A(\infty)-D(r)}+\int_{0}^{1}\right) \frac{R_{1}^{2}(v)}{\Re^{4}(v+D(r))} \mathrm{d} v \\
& =I_{3}(r)+I_{4}(D(r)),
\end{aligned}
$$

where

$$
I_{3}(r) \stackrel{\text { def }}{=} 16 \int_{1}^{A(\infty)-D(r)} \frac{R_{1}^{2}(v)}{\Re^{4}(v+D(r))} \mathrm{d} v \quad \text { is a bounded term }
$$

when $r$ tends to infinity (recalling that $A(\infty)$ is the last exit time of $\Re$ from 2 ), and for small $\delta>0$,

$$
\begin{aligned}
I_{4}(\delta) & \stackrel{\text { def }}{=} 16 \int_{0}^{1} \frac{R_{1}^{2}(v)}{\Re^{4}(v+\delta)} \mathrm{d} v \\
& =16 \delta \int_{0}^{1 / \delta} \frac{R_{1}^{2}(u \delta)}{\Re^{4}((1+u) \delta)} \mathrm{d} u \\
& \stackrel{\text { law }}{=} 16 \int_{0}^{1 / \delta} \frac{R_{1}^{2}(u)}{\Re^{4}(1+u)} \mathrm{d} u .
\end{aligned}
$$

Observe that for any fixed $\nu>0, \Re(1+u) / R(u)=1+o\left(u^{1 / 2-\nu}\right)$ almost surely (as $u \rightarrow \infty$ ). Applying Proposition 2.1 to $I_{4}(\delta)$ (with $d_{1}=2$ and $d_{2}=2+2 \kappa$ ), assembling (3.4), (3.7) and (3.10)-(3.12), and using the monotonicity of $r \mapsto I_{1}(r)+$ $I_{2}(r)$, we readily get $(1.7)-(1.8)$ and (1.10)-(1.12), with

$$
c_{1}=8 \psi(\kappa) \text {. }
$$

This completes the proof of Theorem 1.1.

Remark 3.1. The value of $c_{1}$ found by Kawazu and Tanaka [19] is implicit. The form (3.13) is new. 


\section{Principal VAlues and stable LÉvy processes}

Let $\beta$ be a standard Brownian motion. Its local time is denoted by $L$, and inverse local time at 0 by $\tau$. It has been remarked in Biane and Yor [3] that all possible stable Lévy processes may be obtained as suitable combinations of principal values of Brownian local times, taken at $\{\tau(\lambda) ; \lambda \geq 0\}$. However, the actual computations are made precisely only in the case of the stable index $\alpha=1$.

Since the realization of $\mathcal{S}_{\alpha}^{\mathrm{ca}}$, for $\alpha=1 /(1-q)(0<q<1 / 2)$, given in $(2.18)$ plays an important role in our paper, it seems worth completing the discussion of Biane and Yor [3] on this occasion, and to show directly that the main features of $\mathcal{S}_{\alpha}^{c a}$ may be derived from the principal value representation.

From the canonical decomposition of the Dirichlet process of $\left(\beta^{+}(t)\right)^{1-q}$ (cf., for example Yor [36, p. 3]), we deduce that

$$
\frac{q}{2} \int_{0}^{\infty} \frac{\mathrm{d} x}{x^{1+q}}\left(L_{\tau(\lambda)}^{x}-\lambda\right)=\int_{0}^{\tau(\lambda)} \mathbf{1}_{\{\beta(s)>0\}} \frac{\mathrm{d} \beta(s)}{\beta^{q}(s)} .
$$

Hence, if we write

$$
X^{ \pm}(\lambda) \stackrel{\text { def }}{=} \int_{0}^{\infty} \frac{\mathrm{d} x}{x^{1+q}}\left(L_{\tau(\lambda)}^{ \pm x}-\lambda\right),
$$

and if we can show that $\left\{X^{+}(\lambda) ; \lambda \geq 0\right\}$ and $\left\{X^{-}(\lambda) ; \lambda \geq 0\right\}$ are two independent, completely asymmetric stable processes of index $\alpha=1 /(1-q) \in(1,2)$, then we will have proved (2.18).

The independence follows from excursion theory, while the stability follows from the scaling property of Brownian motion. That $X^{+}$and $X^{-}$are completely asymmetric is due to the fact that they have no negative jumps. Thus (cf. Bertoin [2, p. 217]), there exists a constant $c_{11}=c_{11}(\alpha)$ such that for all $t \in \mathbf{R}$,

$$
\mathbf{E} \exp \left(i t X^{+}(\lambda)\right)=\exp \left[-\lambda c_{11}|t|^{\alpha}\left(1-i \operatorname{sgn}(t) \tan \left(\frac{\pi \alpha}{2}\right)\right)\right] .
$$

From the independence of $X^{+}$and $X^{-}$, we deduce that

$$
\mathbf{E} \exp \left(i t\left(X^{+}(\lambda)-X^{-}(\lambda)\right)\right)=\exp \left(-2 \lambda c_{11}|t|^{\alpha}\right),
$$

which we now compare with the computations of Biane and Yor [3]; this yields $c_{11}=\left(2 \alpha^{2} \psi(\alpha)\right)^{\alpha} / \alpha^{2}$.

We now complete the discussion with the following estimate which is well known in the literature (cf. Bertoin [2, p. 221]).

Lemma 4.1. For each $\lambda>0$,

$$
\mathbf{E}\left[\left|X^{+}(\lambda)\right|^{a}\right]<\infty \quad \text { if and only if } \quad a<\frac{1}{1-q} .
$$

The proof of Lemma 4.1 relies on some well known results on Brownian motion and Bessel processes. Throughout this section,

$$
\begin{aligned}
& \{U(t) ; t \geq 0\} \stackrel{\text { def }}{=} \text { Bessel process of dimension } 0 \text {, with } U(0)=1, \\
& \zeta \stackrel{\text { def }}{=} \inf \{t>0: U(t)=0\}, \\
& \{R(t) ; t \geq 0\} \stackrel{\text { def }}{=} \text { Bessel process of dimension } 4 \text {, with } R(0)=0 \text {, } \\
& \mathbf{L} \stackrel{\text { def }}{=} \sup \{t>0: R(t)=1\} \text {. }
\end{aligned}
$$

In words, $\zeta$ denotes the life time of $U$, and $\mathbf{L}$ the Last exit time of $R$ from 1 . 
Fact 4.2 (Yor [35, p. 52]). Let $\{R(t) ; t \geq 0\}$ and $\mathbf{L}$ be as in (4.3) and (4.4) respectively. Define the normalized Bessel process

$$
\rho(s) \stackrel{\text { def }}{=} \frac{R(s \mathbf{L})}{\sqrt{\mathbf{L}}}, \quad 0 \leq s \leq 1 .
$$

For any bounded functional $F$, we have

$$
\mathbf{E}[F(\rho(s) ; 0 \leq s \leq 1)]=\mathbf{E}\left[\frac{2}{R^{2}(1)} F(R(s) ; 0 \leq s \leq 1)\right] .
$$

Proof of Lemma 4.1. By the second Ray-Knight theorem, $\left\{L_{\tau(\lambda)}^{x} ; x \geq 0\right\}$ is a $\operatorname{BESQ}(0)$, starting from $\lambda$; thus, in the notation of (4.1)-(4.2), it is distributed as $\left\{\lambda U^{2}(x / \lambda) ; x \geq 0\right\}$. Moreover, $U(x / \lambda)=0$ for all $x \geq \lambda \zeta$. Accordingly,

$$
X^{+}(\lambda) \stackrel{\text { law }}{=} \lambda \int_{0}^{\infty} \frac{U^{2}(x / \lambda)-1}{x^{1+q}} \mathrm{~d} x=\lambda^{1-q} \int_{0}^{\zeta} \frac{U^{2}(t)-1}{t^{1+q}} \mathrm{~d} t .
$$

Recall that $U^{2}(t)=1+2 \int_{0}^{t} U(s) \mathrm{d} B(s)$ for some Brownian motion $B$, and that with probability one, $\lim _{t \rightarrow 0}\left(U^{2}(t)-1\right) / t^{q}=0$ (noting that $q<1 / 2$ ). Hence, by integration by parts, $X^{+}(\lambda)$ is distributed as $\left(2 \lambda^{1-q} / q\right) \int_{0}^{\zeta}\left(U(s) / s^{q}\right) \mathrm{d} B(s)$. Applying the Burkholder-Davis-Gundy inequality yields that, for some finite constant $K(a)$, depending only on the value of $a$,

$$
\mathbf{E}\left[\left|X^{+}(\lambda)\right|^{a}\right] \leq K(a)\left(\frac{\lambda^{1-q}}{q}\right)^{a} \mathbf{E}\left[\left(\int_{0}^{\zeta} \frac{U^{2}(s)}{s^{2 q}} \mathrm{~d} s\right)^{a / 2}\right] .
$$

In the notation of (4.1)-(4.4), and using Williams' time reversal theorem for Bessel processes (cf. for example Revuz and Yor [26, Exercise XI.1.23]), we get

$$
\{U(\zeta-t) ; 0 \leq t \leq \zeta\} \stackrel{\text { law }}{=}\{R(t) ; 0 \leq t \leq \mathbf{L}\} \text {. }
$$

Accordingly,

$$
\int_{0}^{\zeta} \frac{U^{2}(s)}{s^{2 q}} \mathrm{~d} s \leq \frac{\zeta^{1-2 q}}{1-2 q} \sup _{0 \leq t \leq \zeta} U^{2}(t) \stackrel{\operatorname{law}}{=} \frac{\mathbf{L}^{1-2 q}}{1-2 q} \sup _{0 \leq t \leq \mathbf{L}} R^{2}(t) .
$$

Let $\rho$ be as in (4.5). The expression on the right hand side is

$$
\frac{\mathbf{L}^{2(1-q)}}{1-2 q} \sup _{0 \leq s \leq 1} \rho^{2}(s) .
$$

By means of (4.6),

$$
\mathbf{E}\left[\left(\int_{0}^{\zeta} \frac{U^{2}(s)}{s^{2 q}} \mathrm{~d} s\right)^{a / 2}\right] \leq \frac{2}{(1-2 q)^{a / 2}} \mathbf{E}\left[R^{-2-2 a(1-q)}(1) \sup _{0 \leq s \leq 1} R^{a}(s)\right] .
$$

Since $\mathbf{E}\left(R^{b}(1)\right)<\infty$ for all $b>-4$ and $\mathbf{E}\left[\sup _{0 \leq s \leq 1} R^{b}(s)\right]<\infty$ for all $b \geq 0$, it follows from Hölder's inequality that the expectation term on the right hand side is finite as long as $2+2 a(1-q)<4$, which means $a<1 /(1-q)$. In view of (4.8), we have proved the "if" part of Lemma 4.1.

To check the "only if" part, let us go back to (4.7) and use (4.9) to arrive at

$$
X^{+}(\lambda) \stackrel{\text { law }}{=} \lambda^{1-q} \int_{0}^{\mathbf{L}} \frac{R^{2}(t)-1}{(\mathbf{L}-t)^{1+q}} \mathrm{~d} t=(\lambda \mathbf{L})^{1-q} \int_{0}^{1} \frac{\rho^{2}(s)-\mathbf{L}}{(1-s)^{1+q}} \mathrm{~d} s,
$$


where $\rho$ is as in (4.5). Again, we can apply Fact 4.2 to see that (writing $G \stackrel{\text { def }}{=}$ $\int_{0}^{1}\left(\left(R^{2}(s)-R^{2}(1)\right) /(1-s)^{1+q}\right) \mathrm{d} s$ for brevity $)$

$$
\begin{aligned}
\mathbf{E}\left[\left|X^{+}(\lambda)\right|^{a}\right] & =2 \lambda^{a(1-q)} \mathbf{E}\left[R^{-2-2 a(1-q)}(1)|G|^{a}\right] \\
& \geq 2 \lambda^{a(1-q)} \mathbf{E}\left[R^{-2-2 a(1-q)}(1) \mathbf{1}_{\{R(1) \leq 1 / 2\}}|G|^{a}\right] .
\end{aligned}
$$

Now take $a=1 /(1-q)$ and consider the function

$$
\varphi(y) \stackrel{\text { def }}{=} \mathbf{E}\left(|G|^{1 /(1-q)} \mid R(1)=y\right), \quad 0 \leq y \leq 1 / 2 .
$$

It is easily seen using the Bessel bridge that $\varphi$ is a continuous and (strictly) positive function on $[0,1 / 2]$, which yields $\inf _{0 \leq y \leq 1 / 2} \varphi(y) \geq c>0$ for some constant $c$ depending on $q$. As a consequence,

$$
\mathbf{E}\left[\left|X^{+}(\lambda)\right|^{1 /(1-q)}\right] \geq 2 c \lambda \mathbf{E}\left[R^{-4}(1) \mathbf{1}_{\{R(1) \leq 1 / 2\}}\right]=\infty,
$$

completing the proof of the "only if" part of Lemma 4.1.

\section{REFERENCES}

[1] Alili, S.: Asymptotic behaviour for random walks in random environments. (preprint)

[2] Bertoin, J.: Lévy Processes. Cambridge University Press, Cambridge, 1996. MR 98e:60117

[3] Biane, P. and Yor, M.: Valeurs principales associées aux temps locaux browniens. Bull. Sci. Math. 111 (1987) 23-101. MR 88g:60188

[4] Bouchaud, J.-P., Comtet, A., Georges, A. and Le Doussel, P.: Classical diffusion of a particle in a one-dimensional random force field. Ann. Phys. 201 (1990) 285-341. MR 91k:82047

[5] Brox, T.: A one-dimensional diffusion process in a Wiener medium. Ann. Probab. 14 (1986) 1206-1218. MR 88f:60132

[6] Carmona, P.: The mean velocity of a Brownian motion in a random Lévy potential. Ann. Probab. $\underline{25}$ (1997) 1774-1788. MR 99b:60126

[7] Comets, F., Menshikov, M.V. and Popov, S.Yu.: Lyapunov functions for random walks and strings in random environment. Ann. Probab. 26 (1998) 1433-1445. CMP 99:09

[8] Csörgö, M., Horváth, L., and Révész, P.: Stability and instability of local time of random walk in random environment. Stoch. Proc. Appl. $\underline{25}$ (1987) 185-202. MR 89c:60078

[9] Deheuvels, P. and Révész, P.: Simple random walk on the line in random environment. Probab. Th. Rel. Fields $\underline{72}$ (1986) 215-230. MR 87m:60193

[10] Dembo, A., Peres, Y. and Zeitouni, O.: Tail estimates for one-dimensional random walk in random environment. Comm. Math. Phys. 181 (1996) 667-683. MR 97h:60074

[11] Dvoretzky, A. and Erdős, P.: Some problems on random walk in space. Proc. 2nd Berkeley Symp. Math. Statist. Probab. 353-367, University of California Press, Berkeley, 1951. MR 13:852b

[12] Gantert, N. and Zeitouni, O.: Quenched sub-exponential tail estimates for onedimensional random walk in random environment. Comm. Math. Phys. 194 (1998) 177-190. CMP 98:14

[13] Greven, A. and den Hollander, F.: Large deviations for a random walk in random environment. Ann. Probab. 22 (1994) 1381-1428. MR 95m:60101

[14] Hu, Y. and Shi, Z.: The local time of simple random walk in random environment. J. Theoretical Probab. 11 (1998) 765-793. CMP 98:15

[15] Hu, Y. and Shi, Z.: The limits of Sinai's simple random walk in random environment. Ann. Probab. $\underline{26}$ (1998) 1477-1521. CMP 99:09

[16] Hughes, B.D.: Random Walks and Random Environments. Vol. II: Random Environments. Oxford Science Publications, Oxford, 1996. MR 98d:60139

[17] Itô, K. and McKean, H.P.: Diffusion Processes and Their Sample Paths. Springer, Berlin, 1965. MR 33:8031

[18] Karlin, S. and Taylor, H.M.: A Second Course in Stochastic Processes. Academic Press, New York, 1981. MR 82j:60003 
[19] Kawazu, K. and Tanaka, H.: A diffusion process in a Brownian environment with drift. J. Math. Soc. Japan 49 (1997) 189-211. MR 99c:60170

[20] Kawazu, K. and Tanaka, H.: Invariance principle for a Brownian motion with large drift in a white noise environment. Hiroshima Math. J. 28 (1998) 129-137.

[21] Kesten, H., Kozlov, M.V. and Spitzer, F.: A limit law for random walk in a random environment. Compositio Math. $\underline{30}$ (1975) 145-168. MR 52:1895

[22] McKean, H.P.: A Hölder condition for Brownian local time. J. Math. Kyoto Univ. 1 (1962) 195-201. MR 26:4421

[23] Mathieu, P.: On random perturbations of dynamical systems and diffusions with a Brownian potential in dimension one. Stoch. Proc. Appl. 77 (1998) 53-67. CMP 98:01

[24] Ray, D.: Sojourn times of a diffusion process. Illinois J. Math. $\underline{7}$ (1963) 615-630. MR 27:6306

[25] Révész, P.: Random Walk in Random and Non-Random Environments. World Scienfitic, Singapore, 1990. MR 92c:60096

[26] Revuz, D. and Yor, M.: Continuous Martingales and Brownian Motion. (Second Edition). Springer, Berlin, 1994. MR 95h:60072

[27] Schumacher, S.: Diffusions with random coefficients. Contemp. Math. $\underline{41}$ (1985) 351-356. MR 88k:60045

[28] Shi, Z.: A local time curiosity in random environment. Stoch. Proc. Appl. $\underline{76}$ (1998) 231-250. CMP 98:17

[29] Sinai, Ya.G.: The limiting behavior of a one-dimensional random walk in a random medium. Th. Probab. Appl. 27 (1982) 256-268. MR 83k:60078

[30] Solomon, F.: Random walks in a random environment. Ann. Probab. $\underline{3}$ (1975) 1-31. MR 50:14943

[31] Tanaka, H.: Diffusion processes in random environments. Proc. ICM (S.D. Chatterji, ed.) 1047-1054. Birkhäuser, Basel, 1995. MR 97g:60112

[32] Tanaka, H.: Limit theorems for a Brownian motion with drift in a white noise environment. Chaos Solitons Fractals 11 (1997) 1807-1816. MR 99a:60085

[33] Warren, J. and Yor, M.: Skew-products involving Bessel and Jacobi processes. Technical report, Department of Mathematics, University of Bath, 1997. To appear in: Séminaire de Probabilités XXXIII. Lecture Notes in Mathematics, Springer, 1999.

[34] Yor, M.: Sur certaines fonctionnelles exponentielles du mouvement brownien réel. J. Appl. Probab. 29 (1992) 202-208. MR 93g:60179

[35] Yor, M.: Local Times and Excursions for Brownian Motion: A Concise Introduction. Lecciones en Matemáticas, Universidad Central de Venezuela, 1995.

[36] Yor, M.: Some Aspects of Brownian Motion. Part II: Some Recent Martingale Problems. Birkhäuser, Basel, 1997. MR 98e:60140

Laboratoire de Probabilités, Université Paris Vi, 4 Place Jussieu, 75252 Paris Cedex 05, France

E-mail address: hu@proba.jussieu.fr

E-mail address: shi@ccr.jussieu.fr

E-mail address: secret@proba.jussieu.fr 